\title{
Second-harmonic imaging of semiconductor quantum dots
}

Østergaard, John Erland; Bozhevolnyi, Sergey I.; Pedersen, Kjeld; Jensen, Jacob Riis; Hvam, Jørn Märcher

Published in:

Applied Physics Letters

Link to article, DOI:

10.1063/1.1306634

Publication date:

2000

Document Version

Publisher's PDF, also known as Version of record

Link back to DTU Orbit

Citation (APA):

Østergaard, J. E., Bozhevolnyi, S. I., Pedersen, K., Jensen, J. R., \& Hvam, J. M. (2000). Second-harmonic imaging of semiconductor quantum dots. Applied Physics Letters, 77(6), 806-808.

https://doi.org/10.1063/1.1306634

\section{General rights}

Copyright and moral rights for the publications made accessible in the public portal are retained by the authors and/or other copyright owners and it is a condition of accessing publications that users recognise and abide by the legal requirements associated with these rights.

- Users may download and print one copy of any publication from the public portal for the purpose of private study or research.

- You may not further distribute the material or use it for any profit-making activity or commercial gain

- You may freely distribute the URL identifying the publication in the public portal

If you believe that this document breaches copyright please contact us providing details, and we will remove access to the work immediately and investigate your claim 


\title{
Second-harmonic imaging of semiconductor quantum dots
}

\author{
John Erland ${ }^{\mathrm{a})}$ \\ Research Center COM, Technical University of Denmark, Building 349, DK-2800 Lyngby, Denmark \\ Sergey I. Bozhevolnyi and Kjeld Pedersen \\ Institute of Physics, Aalborg University, Pontoppidanstrcede 103, DK-9220 Aalborg, Denmark \\ Jacob R. Jensen and Jørn M. Hvam \\ Research Center COM, Technical University of Denmark, Building 349, DK-2800 Lyngby, Denmark
}

(Received 30 March 2000; accepted for publication 12 June 2000)

\begin{abstract}
Resonant second-harmonic generation is observed at room temperature in reflection from self-assembled InAlGaAs quantum dots grown on a GaAs (001) substrate. The detected second-harmonic signal peaks at a pump wavelength of $\sim 885 \mathrm{~nm}$ corresponding to the quantum-dot photoluminescence maximum. In addition, the second-harmonic spectrum exhibits another smaller but well-pronounced peak at $765 \mathrm{~nm}$ not found in the linear experiments. We attribute this peak to the generation of second-harmonic radiation in the AlGaAs spacer layer enhanced by the local symmetry at the quantum-dot interface. We further observe that second-harmonic images of the quantum-dot surface structure show wavelength-dependent spatial variations. Imaging at different wavelength is used to demonstrate second-harmonic generation from the semiconductor quantum dots. (C) 2000 American Institute of Physics. [S0003-6951(00)00932-3]
\end{abstract}

Second-harmonic generation (SHG) is extremely sensitive to the symmetry of materials ${ }^{1}$ and is widely used as a nondestructive and noncontact probe of surfaces and interfaces. ${ }^{2}$ Furthermore, starting with the first experiments on spatially resolved SHG from a dye monolayer, ${ }^{3}$ SHG microscopy has appeared as a very useful characterization tool for inhomogeneous surfaces. It has recently been applied to imaging of periodically poled ferroelectric domains, ${ }^{4}$ poled silica waveguides, ${ }^{5}$ and domain walls,${ }^{6}$ domain structures in epitaxially grown magnetic garnet films ${ }^{7}$ and polymer monolayers, ${ }^{8}$ and polar orientational distribution in thin polymer films. ${ }^{9}$ Semiconductor quantum dots (QDs), whose unique properties promise wide applications in optoelectronics, ${ }^{10}$ is yet another interesting object for SHG microscopy. The electronic eigenstates of the QDs are strongly influenced by their sizes and shapes as well as by strain and piezoelectric fields. ${ }^{11}$ Self-assembled InAs/GaAs QDs have been extensively studied with various methods, ${ }^{10}$ however, the wealth of information encoded in the nonlinear optical coefficients is still largely unexplored. As far as SHG is concerned, the main problem is related to the fact that GaAs possesses very large second-order susceptibilities, ${ }^{12}$ so that any second harmonic $(\mathrm{SH})$ radiation from the QDs has to be distinguished from the $\mathrm{SH}$ generated in GaAs. Our idea is to use SHG in configurations where the bulk and surface contributions are forbidden for a homogeneous sample, so that the only source of SHG is associated with nanostructures embedded in the host material. ${ }^{13}$

In this letter, we demonstrate that, for normal incidence of resonant pump radiation, SHG in reflection from a sample containing selfassembled $\mathrm{In}_{0.50} \mathrm{Al}_{0.08} \mathrm{Ga}_{0.42} \mathrm{As}$ QDs grown on a GaAs (001) substrate is dominated by the SH radiation

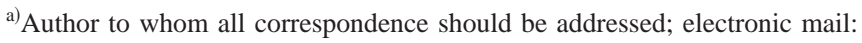
jeo@com.dtu.dk
}

from the QDs. The spectral analysis shows correspondence to the energy eigenstates characterized by photoluminescence (PL) measurements. The wavelength dependence of the SH signal is found to exhibit another peak at $765 \mathrm{~nm}$, which is related to the interface between the QD layer and the AlGaAs spacer layer. Furthermore, we present preliminary results on wavelength resolved SH imaging of QDs conducted for different linear polarizations of the SH radiation. Transformation of the SH images when changing the pump wavelength is observed and attributed to the spatially varying conditions for SHG generation at the inhomogeneous surface and therefore essential for the identification of the $\mathrm{SH}$ radiation from the QDs.

The QD sample used in this work was fabricated by molecular beam epitaxy, where 6 monolayers of $\mathrm{In}_{0.50} \mathrm{Al}_{0.08} \mathrm{Ga}_{0.42} \mathrm{As}$ were grown with 100 (20) $\mathrm{nm}$ $\mathrm{Al}_{0.16} \mathrm{Ga}_{0.84} \mathrm{As}$ bottom (top) spacer layer and $8 \mathrm{~nm}$ $\mathrm{Al}_{0.40} \mathrm{Ga}_{0.60}$ As barriers terminated with a GaAs cap layer of $20 \mathrm{~nm}$. The molefractions of the QD material was chosen to fit the energies of the QD states into a wavelength range accessible with the Ti:sapphire laser used for the SHG, see the following. The Stranski-Krastanow formation of the QDs was evident from the reflection high energy electron diffraction spectrum during growth as well as from atomic force microscope studies of uncapped QD samples grown under similar conditions indicating typical QD distances of $50 \mathrm{~nm}$. To remove possible influences from the semiinsulating (001) GaAs substrate on the SHG experiments, a $500 \mathrm{~nm}$ GaAs buffer layer was grown on top of an etch layer of $50 \mathrm{~nm}$ AlAs. After etching with $10 \%$ hydroflouric acid and lift-off using a wax, the QD sample was mounted on a high optical quality sapphire substrate using only van der Waals forces to avoid any additional strain in the sample.

The mounted QD sample was characterized by measuring the PL spectrum at room temperature [Fig. 1(a)]. The PL spectrum, after excitation with a $\mathrm{HeNe}$ laser over two de- 


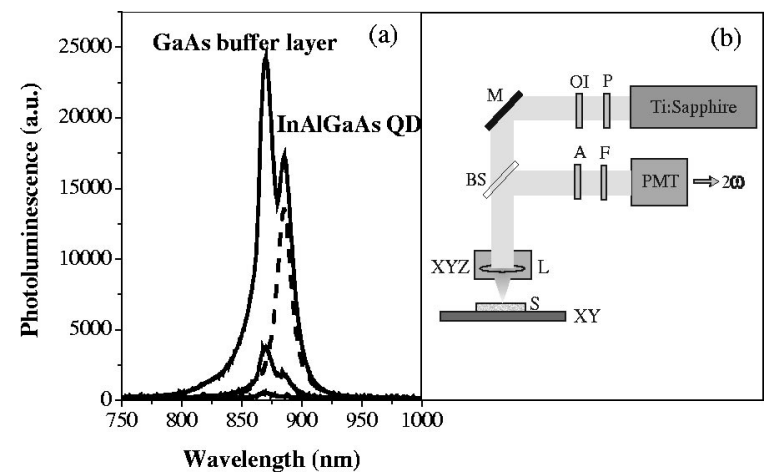

FIG. 1. (a) Photoluminescence characterization of the QD sample for three different $\mathrm{HeNe}(632.8 \mathrm{~nm})$ laser light intensities (solid) revealing the GaAs buffer layer at $870 \mathrm{~nm}$ and the QD resonance at the design wavelength of $885 \mathrm{~nm}$. The extracted QD resonance with a linewidth of $15 \mathrm{~nm}$ from line shape analysis is shown as a dashed line. (b) The second-harmonic imaging setup in reflection with a PMT: photomultiplier, BS: beam splitter, S: sample, M: mirror, L: focusing lense, OI: optical isolator, F: filter, A: analyzer, and P: polarizer.

cades of $\mathrm{cw}$ power up to $1 \mathrm{~mW}$ focused to a spot of $\sim 40 \mu \mathrm{m}$, shows two strong resonances: the PL from the GaAs buffer layer at $870 \mathrm{~nm}(1.42 \mathrm{eV})$ and from the QDs at the design wavelength of $885 \mathrm{~nm}(1.40 \mathrm{eV})$. From the spectra we estimate an inhomogeneous broadening of the QDs of $15 \mathrm{~nm}(24 \mathrm{meV})$, i.e., the QD eigenstates are well separated from the GaAs absorption band edge. The InAlGaAs wetting layer resonance appeared as a small shoulder on the GaAs PL signal at $\sim 810 \mathrm{~nm}$, but was not directly observable in the SHG experiments.

The experimental setup for SHG microscopy represents a scanning optical microscope in reflection geometry [Fig. 1(b)]. The linearly polarized light beam from a mode-locked Ti-sapphire laser $\left(P_{\omega}^{\mathrm{av}} \approx 50 \mathrm{~mW}\right.$, repetition rate $f \approx 80 \mathrm{MHz}$, pulse duration $\tau \sim 200 \mathrm{fs}$ ) was focused on the sample surface at normal incidence with a $\times 40$ microscope objective (the spot size $\sim 2 \mu \mathrm{m}$ ), and the sample was scanned along the surface plane by a stepper-motor driven $x y$ stage. The reflected SH radiation was detected with a photomultiplier, whose output was recorded as a function of the scanning coordinate (with the step length of $0.25 \mu \mathrm{m}$ ). Note that only at normal incidence is the SHG from the bulk and the surface forbidden by symmetry. Our first measurements in GaAs showed that the SHG in reflection (at carefully adjusted normal incidence) from a (001) substrate was negligibly small, and the SH signal was more than three orders of magnitude weaker than that from a (111) substrate. ${ }^{13}$ We have used an optical isolator to avoid the reflected pump beam coupling back into the laser cavity [Fig. 1(b)]. The incident pump beam was linearly polarized either along the $x$ or $y$ axis in the experimental setup, and we detected the $\mathrm{SH}$ radiation at two orthogonal linear polarizations $x$ and $y$. Because of the lift-off of the sample, the crystal axis of the sample was unknown. However, in the investigations of the SH signals for the four different combinations of linear polarizations between the incident pump and the detected SHG signal, the SH signal in the $y y$ configuration was on average one order of magnitude larger than that in the other combinations (data not shown). With the investigated QD sample and the polarization configuration with the best SHG yield, a resonant pump power of about $50 \mathrm{~mW}$ at the sample resulted in $\mathrm{SH}$ Downloaded 05 Feb 2010 to 192.38.67.112. Redistribution subject
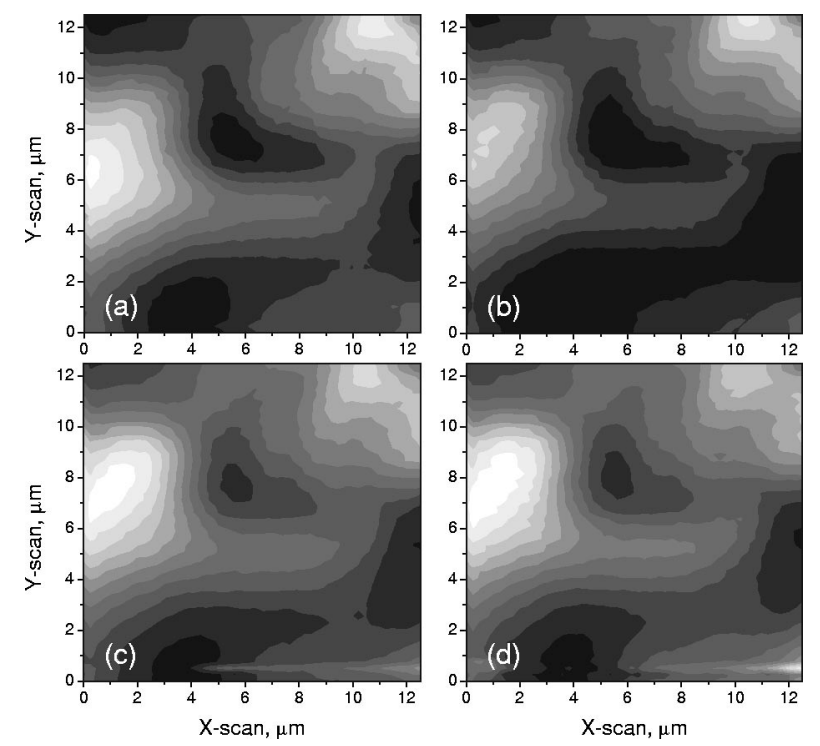

FIG. 2. Room temperature second-harmonic images recorded at pump wavelengths of: (a) $896 \mathrm{~nm}$, (b) $885 \mathrm{~nm}$, (c) $879 \mathrm{~nm}$, and (d) $875 \mathrm{~nm}$.

signals in the range $10^{3}-10^{4}$ counts/s. This signal level allowed us to use a count time of $0.1 \mathrm{~s}$ and to record $\mathrm{SH}$ images containing $100 \times 100$ points within $\sim 16 \mathrm{~min}$.

The main features observed in our experiment are (i) the spatial dependence of the SH signal, (ii) the transformation of the SH image with the wavelength (Fig. 2), and (iii) the wavelength dependence of the average SH signal (Fig. 3). Strong enhancement of the average SH signal at $\sim 885 \mathrm{~nm}$ with the width of the enhancement $(<20 \mathrm{~nm})$ being comparable to the linear QD PL spectrum is observed [cf. Figs. 3 and 1(a)]. This indicates that the detected SH radiation originates from QDs, whose energy states are inhomogeneously broadened. With the spot size obtainable in far-field microscopy, the incident beam interacts with a large $\left(\sim 10^{4}\right)$ number of QDs and the SH signal averages out the responses of individual QDs. However, we have observed that the SH images show strong variation in the SH signal (Fig. 2). This feature can be attributed to variations mainly due to the local

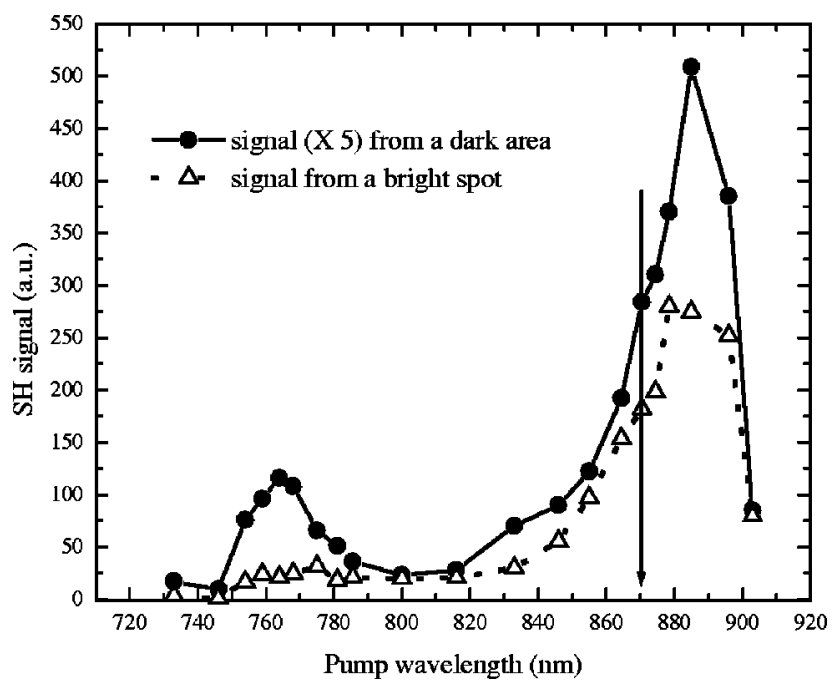

FIG. 3. The spectral changes of the SHG signal recorded at the darker spot (open circles) in the center (dark area) and at the brighter spot to the left (open triangles) of the images in Fig. 2. The vertical arrow indicates the absorption band edge for GaAs at $870 \mathrm{~nm}$. 
surface orientation since the large number of QDs average their spatial variation and their individual optical response. Since the positions of bright and dark spots were found to be stable with respect to the pump wavelength, we believe that bright spots represent the areas that are locally tilted with respect to the average surface plane. Such a tilt would break the symmetry and allow SHG from the bulk, i.e., from the GaAs buffer layer. Therefore, one should expect that the $\mathrm{SH}$ signal from bright spots may contain a substantial contribution from the bulk SHG. Indeed, the main peak in the SHG spectrum obtained at the bright spot is shifted toward lower wavelengths (in comparison with the peak from the dark spot) and exhibits a pronounced shoulder at the wavelength of $870 \mathrm{~nm}$ corresponding to the maximum of PL from the GaAs buffer layer (Fig. 3). Actually, a similar (but less pronounced) shoulder is also seen in the main peak obtained at the dark spot indicating that, even at normal incidence, there is a (relatively weak) bulk contribution to SHG. This contribution can be accounted for by the fact that the pump light is scattered by the embedded nanostructures so that the local normal incidence condition is not fulfilled for the scattered light. The same reasoning can be used to explain a second peak in the SHG spectrum at $765 \mathrm{~nm}$ (Fig. 3), which corresponds to the room temperature band gap of the $\mathrm{Al}_{0.16} \mathrm{Ga}_{0.84} \mathrm{As}$ spacer layer. ${ }^{14}$ Finally, let us notice that the shape of the dark spot depends on the pump wavelength (Fig. 2). We believe that this modification can be related to the interplay between the SHG response from the dark and bright areas with different spatial and spectral SHG response. The observed features demonstrate that the wavelength resolved SHG microscopy is a very sensitive tool for investigation of nanostructured semiconductors.

In conclusion, we have demonstrated SHG from semiconductor QDs at room temperature using resonant sample illumination at normal incidence. In this configuration, the second-order nonlinear optical susceptibility $\chi^{(2)}$ due to bulk and surface contributions from the different semiconductor layers composing the structure is ideally zero. The SHG spectrum is peaked at $885 \mathrm{~nm}$ in accordance with the InAlGaAs QD spectrum of the PL. Scanning over the sample surface was used to map the strength of SHG over a surface area of $12.5 \times 12.5 \mu \mathrm{m}^{2}$. The $\mathrm{SH}$ imaging was used to separate the $\mathrm{SH}$ signals from the QDs and the GaAs bulk, whose contribution notably increases when the normal incidence condition deteriorates. This furthermore enabled the observation of a second peak in the SHG spectrum at $765 \mathrm{~nm}$ resulting from light scattering at the interface between the QD layer and the AlGaAs spacer layer. Finally, we would like to stress that, contrary to the PL characterization technique, the SHG microscopy in reflection used in this work is inherently insensitive to the presence of a GaAs substrate even at room temperature. It can therefore be successfully used at room temperature to study embedded nanostructures without etching away a substrate. ${ }^{13}$ This is normally a complicated procedure which makes subsequent characterization, e.g., determination of the orientation of the crystal axis, very difficult because of reduced sample quality. We believe that further exploration of this technique would enable us to investigate various fascinating phenomena such as the influence of strain and piezoelectric fields on the QD characteristics. ${ }^{11}$

The authors gratefully acknowledge financial support from the Danish Natural Science Research Council under Contracts 9901971 and 9903131.

${ }^{1}$ R. W. Boyd, Nonlinear Optics (Academic, London, 1992).

${ }^{2}$ T. F. Heinz, in Nonlinear Surface Electromagnetic Phenomena, edited by H. Ponath and G. Stegeman (Elsevier, Amsterdam, 1991), p. 353.

${ }^{3}$ G. T. Boyd, Y. R. Shen, and T. W. Hänsch, Opt. Lett. 11, 97 (1986).

${ }^{4}$ S. Kurimura and Y. Uesu, J. Appl. Phys. 81, 369 (1997); Y. Uesu, S. Kurimura, and Y. Yamamoto, Appl. Phys. Lett. 66, 2165 (1995).

${ }^{5}$ J. Arentoft, K. Pedersen, S. I. Bozhevolnyi, M. Kristensen, P. Yu, and C. B. Nielsen, Appl. Phys. Lett. 76, 25 (2000).

${ }^{6}$ S. I. Bozhevolnyi, J. M. Hvam, K. Pedersen, F. Laurell, H. Karlsson, T. Skettrup, and M. Belmonte, Appl. Phys. Lett. 73, 1814 (1998).

${ }^{7}$ V. Kirilyuk, A. Kirilyuk, and Th. Rasing, Appl. Phys. Lett. 70, 2306 (1997)

${ }^{8}$ M. Flörsheimer, Phys. Status Solidi A 173, 15 (1999).

${ }^{9}$ J. Vidra and M. Eich, Appl. Phys. Lett. 72, 275 (1998).

${ }^{10}$ D. Bimberg, M. Grundmann, and N. N. Ledentsov, Quantum Dot Heterostructures (Wiley, Chichester, 1998).

${ }^{11}$ O. Stier, M. Grundmann, and D. Bimberg, Phys. Rev. B 59, 5688 (1999).

${ }^{12}$ D. N. Nikogosyan, Properties of Optical and Laser-Related Materials: A Handbook (Wiley, Chichester, 1997).

${ }^{13}$ J. Erland, P. Yu, S. I. Bozhevolnyi, J. M. Hvam, and N. N. Ledentsov, Technical Digest QELS'99 (Baltimore, Maryland, May 1999), p. 233.

${ }^{14}$ L. Pavesi and M. Guzzi, J. Appl. Phys. 75, 4779 (1994). 